OPEN ACCESS

Edited by:

Triantafyllos Stylianopoulos, University of Cyprus, Cyprus

Reviewed by: Yong Chen,

Fudan University, China Jianling Bi,

The University of lowa, United States

*Correspondence: Jilong Yang yangjilong@tjmuch.com

${ }^{+}$These authors have contributed equally to this work

Specialty section: This article was submitted to Cancer Molecular Targets and Therapeutics, a section of the journal Frontiers in Oncology

Received: 21 September 2021 Accepted: 08 December 2021 Published: 03 January 2022

Citation:

Liao Z, Zhang C, Yang T, Liu H, Yang $S, L i T$, Xing $R$, Teng $S$, Yang $Y$,

Zhao J, Zhao G, Bai X, Zhu L and Yang J (2022) Chemotherapy Combined With Recombinant Human Endostatin (Endostar)

Significantly Improves the

Progression-Free Survival of Stage IV Soft Tissue Sarcomas.

Front. Oncol. 11:778774. doi: 10.3389/fonc.2021.778774

\title{
Chemotherapy Combined With Recombinant Human Endostatin (Endostar) Significantly Improves the Progression-Free Survival of Stage IV Soft Tissue Sarcomas
}

\begin{abstract}
Zhichao Liao ${ }^{1,2 \dagger}$, Chao Zhang ${ }^{1,2 \dagger}$, Tielong Yang ${ }^{1,2}$, Haotian Liu ${ }^{1,2}$, Songwei Yang ${ }^{1,3}$, Ting $\mathrm{Li}^{1,2}$, Ruwei Xing ${ }^{1,2}$, Sheng Teng ${ }^{1,2}$, Yun Yang ${ }^{1,2}$, Jun Zhao ${ }^{1,2}$, Gang Zhao ${ }^{2,4}$, $\mathrm{Xu} \mathrm{Bai}^{2,5}$, Lei Zhu ${ }^{2,6}$ and Jilong Yang ${ }^{1,2 *}$
\end{abstract}

\begin{abstract}
${ }^{1}$ Department of Bone and Soft Tissue Tumor, Tianjin Medical University Cancer Institute and Hospital, Tianjin, China, ${ }^{2}$ National Clinical Research Center for Cancer, Key Laboratory of Cancer Prevention and Therapy, Tianjin's Clinical Research Center for Cancer, Tianjin Medical University Cancer Institute and Hospital, Tianjin, China, ${ }^{3}$ Departments of Bone and Soft Tissue Tumor, Chongqing University Cancer Hospital, Chongqing, China, ${ }^{4}$ Department of Pathology, Tianjin Medical University Cancer Institute and Hospital, Tianjin, China, ${ }^{5}$ Department of Radiology, Tianjin Medical University Cancer Institute and Hospital, Tianjin, China, ${ }^{6}$ Department of Molecular Imaging and Nuclear Medicine, National Clinical Research Center for Cancer, Tianjin Medical University Cancer Institute and Hospital, Tianjin, China
\end{abstract}

Purpose: Our previously study showed that recombinant human endostatin (Endostar) combined with chemotherapy had significant activity to increase the mPFS in patients with advanced sarcomas with tolerable side effects. However, the small cohort size and short follow-up time made it difficult to screen sensitive sarcoma subtypes and determine whether there is an overall survival benefit. With the largest sarcoma cohort to our knowledge, we try to confirm the efficacy and safety of chemotherapy combined with Endostar in stage IV sarcomas, with the specific purpose of finding out the sensitive sarcoma types for this combined treatment.

Methods: After the exclusion of ineligible patients, 156 patients with stage IV bone and soft tissue sarcomas were included in this study according to the inclusion criteria.

Results: By the end of follow-up, the ORR was $10.7 \%$ (9/84) vs $1.4 \%(1 / 72)$ ( $p=0.041)$, the DCR was $26.2 \%(22 / 84)$ vs $5.6 \%(4 / 72)(p=0.001)$ in the combined group and chemotherapy group, respectively. The mPFS of combined group was significantly longer than the chemotherapy group (10.42 vs 6.87 months, $p=0.003$ ). The mOS were 26.84 months and 23.56 months, without significant difference $(p=0.481)$. In osteogenic sarcoma, there was no statistically significant difference in the mPFS between the two groups ( $p=0.59$ ), while in the soft tissue sarcoma, the mPFS in the combined group was significantly higher than that of the chemotherapy group (11.27 vs 8.05 months, $p=0.004$ ). Specifically, undifferentiated polymorphic sarcoma (UPS) was the possible sarcoma subtypes that benefited from the combined therapy. For the 38 UPS patients (28 patients in the combined group and 10 patients in the chemotherapy group), the mPFS 
in the combined group was up to 14.88 months, while it was only 7.1 months in the chemotherapy group, with a significant difference $(p=0.006)$. The most common adverse events in the combined group were myelosuppression, gastrointestinal reactions and abnormal liver function, without significant difference in two groups.

Conclusion: Chemotherapy plus Endostar could prolong mPFS and improve ORR and DCR in patients with stage IV soft tissue sarcoma, suggesting that the combined therapy could improve the patient prognosis in soft tissue sarcomas, especially the UPS patients.

Keywords: sarcoma, endostar, chemotherapy, undifferentiated pleomorphic sarcoma, progression-free survival, adverse events

\section{INTRODUCTION}

Sarcomas are highly malignant tumors with complex pathological types and large heterogeneity and are mainly divided into two major categories: osteogenic sarcoma and soft tissue sarcoma, both of which have a poor prognosis (1-3). The median overall survival (mOS) for patients with metastatic soft tissue sarcoma is only 12.8-14.3 months (4). For patients with lung metastases or chemotherapy-resistant osteosarcoma, the 5year survival rate is only $20 \%(5,6)$.

Currently, chemotherapy remains the cornerstone of treatment for patients with advanced unresectable sarcoma. For patients with soft tissue sarcoma, anthracycline-based combination chemotherapy is the first-line chemotherapy regimen. When firstline chemotherapy fails, the options include gemcitabine, docetaxel, ifosfamide, dacarbazine, etc. (4, 7-10). The standard chemotherapy regimens for osteosarcoma patients include high doses of methotrexate, cisplatin, and ifosfamide (5). Unfortunately, despite advances in surgical techniques, the prognosis of patients with bone and soft tissue sarcoma has not improved significantly in recent years. For example, the OS rate for patients with osteosarcoma, which reached $65 \%-70 \%$ in the 1970 s, has not improved significantly over the past three decades and has reached a plateau (11). In recent years, there have been an increasing number of clinical trials evaluating the value of chemotherapy combined with other therapies in patients with sarcoma. The combination of doxorubicin and olaratumab (an inhibitor that blocks the PDGF pathway) significantly prolonged the mOS in a phase II trial but failed to demonstrate this finding in a phase III trial $(12,13)$. A phase II clinical trial showed that paclitaxel combined with bevacizumab was not recommended for the treatment of angiosarcoma due to increased adverse events (14). The combination of chemotherapy agents, such as doxorubicin combined with trabectedin, also does not show any advantage over doxorubicin alone (15). Doxorubicin combined with immunotherapy, such as pembrolizumab, has

Abbreviations: UPS, undifferentiated pleomorphic sarcoma; CR, complete response; $\mathrm{PR}$, partial response; $\mathrm{SD}$, stable disease; $\mathrm{PD}$, progressive disease; $\mathrm{ORR}$, objective response rate; $\mathrm{DCR}$, disease control rate; $\mathrm{mPFS}$, median progression-free survival; mOS, median overall survival; AJCC, American Joint Committee on Cancer; MPNST, malignant peripheral nerve sheath tumor; AI, doxorubicin+ ifosfamide; T10, methotrexate+ cisplatin+ doxorubicin; GT, gemcitabine+ paclitaxel; MAID, mesna + doxorubicin + ifosfamide+ dacarbazine; CAV, cyclophosphamide+ doxorubicin + vincristine; IE, ifosfamide + etoposide. shown promising progression-free survival (PFS) and OS benefits, but further study is needed because a small number of patients was enrolled (16). Therefore, there is an urgent need to find new treatments to improve the chemotherapy sensitivity of patients with advanced sarcoma.

The growth and metastasis of malignant tumors are closely related to angiogenesis, so antiangiogenic therapy is always a hot research spot and direction of tumor treatment (17-19). Endostatin is an endogenous protein that can inhibit the expression of vascular endothelial growth factor (VEGF); therefore, tumor angiogenesis can be inhibited $(20,21)$. However, endostatin is unstable under in vitro conditions (11). Endostar is a safe and well-tolerated recombinant human endostatin that can inhibit the growth and metastasis of tumors (22). Previous clinical trials have demonstrated definitive antitumor activity of Endostar in patients with lung cancer, breast cancer, melanoma and nasopharyngeal carcinoma, with manageable and tolerable adverse events (23-30). Several studies of Endostar in combination with chemotherapy for the treatment of advanced sarcoma have shown encouraging results, one of which was performed at our cancer center $(11,31-$ 33). These results suggested that Endostar combined with chemotherapy may be a promising treatment for patients with advanced sarcoma. However, these studies were limited by small sample sizes and did not screen the pathological subtypes sensitive to Endostar combined with chemotherapy. Therefore, in the present study, we enrolled the largest stage IV sarcoma cohort who received Endostar combined with a chemotherapy regimen and attempted to analyze independent risk factors affecting prognosis. In addition, we screened for the pathological subtypes with the greatest sensitivity to Endostar combined with chemotherapy. The results suggested that stage IV soft tissue sarcomas, especially undifferentiated pleomorphic sarcoma (UPS), might benefit from the combination of Endostar with chemotherapy, which might supply more management choices for patients with advanced sarcoma after more evidence accumulations.

\section{MATERIALS AND METHODS}

\section{Patients and Treatment}

A total of 178 patients with advanced sarcoma who were admitted to the Department of Bone and Soft Tissue Tumor, 
Tianjin Medical University Cancer Institute and Hospital, between July 2009 and September 2020 were included in this study (Figure 1A). All patients were pathologically confirmed to have bone or soft tissue sarcoma, and the pathological types included the most common types of bone and soft tissue sarcoma. Among the patients, 10 had no target lesions or the target lesions could not be measured, 8 received only one cycle of Endostar treatment, and 4 received only one cycle of chemotherapy. These patients were excluded from the final efficacy and adverse event evaluations (Figure 1A). Of the remaining 156 patients, 84 who received Endostar combined with chemotherapy were assigned as the combined group (Figure 1C), and 72 patients who received only chemotherapy (Figure 1D) were assigned as the chemotherapy (control) group. This study of combination of Endostar and chemotherapy complied with the Declaration of Helsinki and was approved by the Ethics Committee of Tianjin Medical University Cancer Institute and Hospital (Ethical batch number, E2017023).

The chemotherapy regimens used in the two groups mainly included AI, T10, GT, MAID, CAV, and IE. Patients with osteosarcoma received T10 as the main chemotherapy method, while patients with soft tissue sarcoma primarily received AI,

A

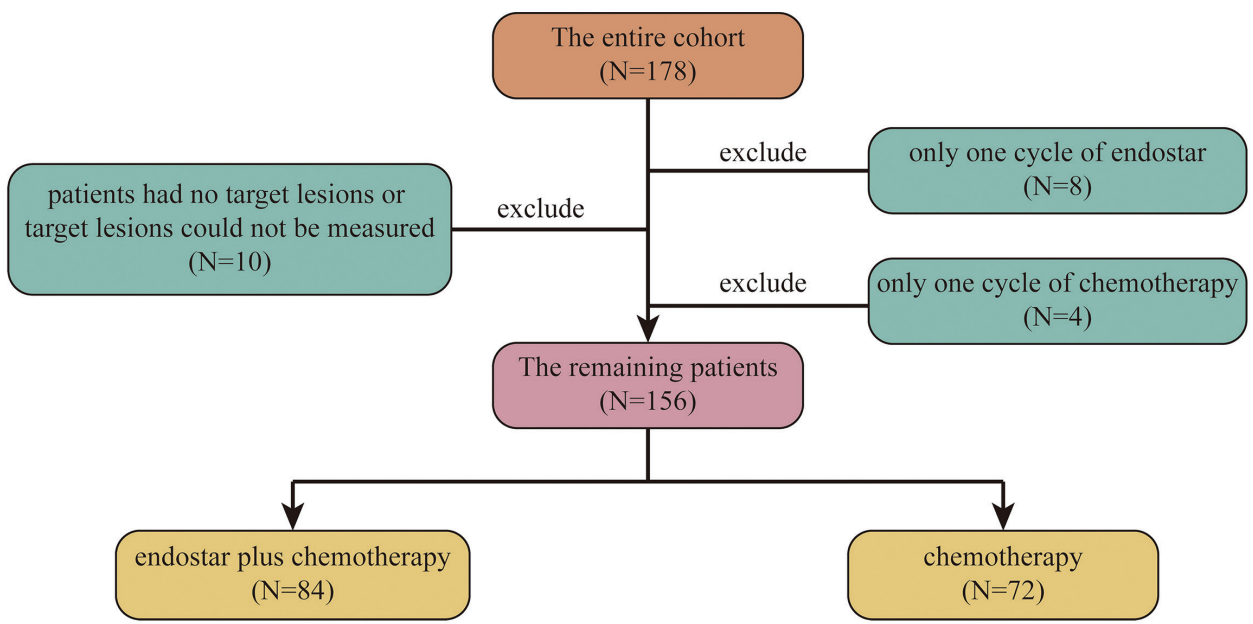

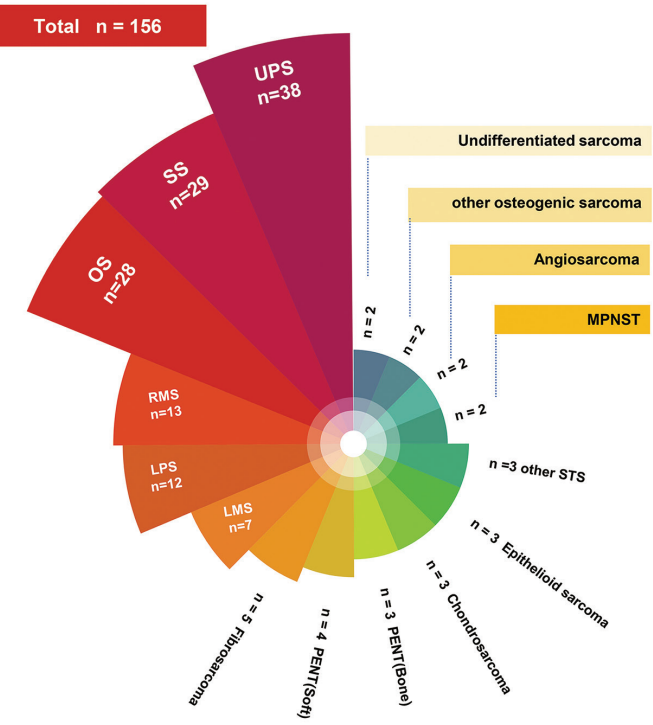

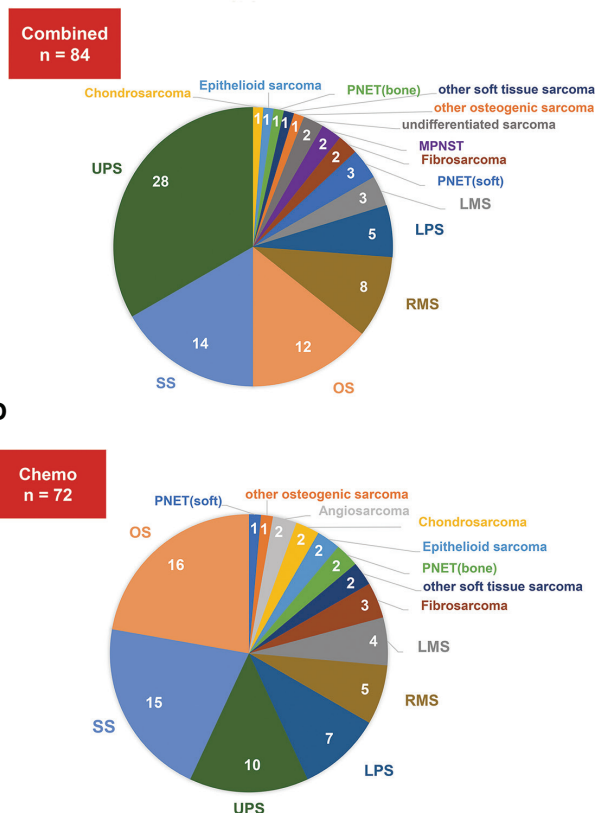

FIGURE 1 | (A) Flow chart for screening patients. (B) Numbers of patients with different pathological subtypes in the entire cohort. (C) Numbers of patients with different pathological subtypes in the Endostar combined with chemotherapy group. (D) Numbers of patients with different pathological subtypes in the traditional chemotherapy group. 
MAID, and GT (Table 1). In the combined group, $15 \mathrm{mg}$ Endostar (dissolved in $500 \mathrm{ml}$ physiological saline) was given per day during chemotherapy, used continuously for 4 hours for 14 consecutive days, and used again after at least 7 days. Each patient's heart rate and blood pressure, blood oxygen saturation and skin allergic reactions were closely monitored during the use of Endostar. All patients in this group received at least two cycles of Endostar treatment.

TABLE 1 | Patients Characteristics.

\begin{tabular}{|c|c|c|c|c|}
\hline & overall & chemotherapy & endostar plus chemotherapy & $p$ value \\
\hline patients(\%) & 156 & 72 & 84 & \\
\hline gender & & & & 0.382 \\
\hline male & $95(60.9)$ & $47(65.3)$ & $48(57.1)$ & \\
\hline female & $61(39.1)$ & $25(34.7)$ & $36(42.9)$ & \\
\hline age median [range] & $45.50[10,81]$ & $44.50[10,81]$ & $48.00[11,72]$ & 0.828 \\
\hline$<60$ years & $120(76.9)$ & $55(76.4)$ & $65(77.4)$ & 0.883 \\
\hline$>=60$ years & $36(23.1)$ & $17(23.6)$ & 19 (22.6) & \\
\hline pathological classification & & & & 0.139 \\
\hline osteogenic sarcoma & $36(23.1)$ & $21(29.2)$ & 15 (17.9) & \\
\hline soft tissue sarcoma & $120(76.9)$ & $51(70.8)$ & $69(82.1)$ & \\
\hline location & & & & 0.227 \\
\hline extremity & $111(71.2)$ & $57(79.2)$ & $54(64.3)$ & \\
\hline trunk & $28(17.9)$ & $10(13.9)$ & $18(21.4)$ & \\
\hline retroperitoneum & $10(6.4)$ & $2(2.8)$ & $8(9.5)$ & \\
\hline head/neck & $3(1.9)$ & $1(1.4)$ & $2(2.4)$ & \\
\hline others & $4(2.6)$ & $2(2.8)$ & $2(2.4)$ & \\
\hline ECOG & & & & 0.146 \\
\hline$<=1$ & $100(64.1)$ & $51(70.8)$ & 49 (58.3) & \\
\hline$>1$ & $56(35.9)$ & $21(29.2)$ & $35(41.7)$ & \\
\hline surgery & & & & 0.76 \\
\hline wide resection & $89(57.1)$ & $45(62.5)$ & $44(52.4)$ & \\
\hline amputation & $19(12.2)$ & $8(11.1)$ & $11(13.1)$ & \\
\hline local resection & $26(16.7)$ & $10(13.9)$ & $16(19.0)$ & \\
\hline slicer biopsy & $11(7.1)$ & $4(5.6)$ & 7 (8.3) & \\
\hline needle aspiration biopsy & $11(7.1)$ & $5(6.9)$ & $6(7.1)$ & \\
\hline metastatic location & & & & 0.311 \\
\hline lung & $90(57.7)$ & $41(56.9)$ & 49 (58.3) & \\
\hline lung plus other site & $21(13.5)$ & 7 (9.7) & $14(16.7)$ & \\
\hline non-lung & $45(28.8)$ & $24(33.3)$ & $21(25.0)$ & \\
\hline radiotherapy & & & & 0.880 \\
\hline no & $92(59.0)$ & $42(58.3)$ & $50(59.5)$ & \\
\hline yes & $64(41.0)$ & $30(41.7)$ & $34(40.5)$ & \\
\hline detailed classification of pathology & & & & $\mathrm{N} / \mathrm{A}$ \\
\hline UPS & $38(24.4)$ & $10(13.9)$ & 28 (33.3) & \\
\hline synovial sarcoma & 29 (18.6) & $15(20.8)$ & $14(16.7)$ & \\
\hline osteosarcoma & 28 (17.9) & $16(22.2)$ & $12(14.3)$ & \\
\hline rhabdomyosarcoma & $13(8.3)$ & $5(6.9)$ & $8(9.5)$ & \\
\hline liposarcoma & $12(7.7)$ & $7(9.7)$ & $5(6.0)$ & \\
\hline leiomyosarcoma & $7(4.5)$ & $4(5.6)$ & $3(3.6)$ & \\
\hline fibrosarcoma & $5(3.2)$ & $3(4.2)$ & $2(2.4)$ & \\
\hline Ewing's sarcoma of soft tissue/PNET & $4(2.6)$ & $1(1.4)$ & $3(3.6)$ & \\
\hline chondrosarcoma & $3(1.9)$ & $2(2.8)$ & $1(1.2)$ & \\
\hline Ewing's sarcoma of bone/PNET & $3(1.9)$ & $2(2.8)$ & $1(1.2)$ & \\
\hline epithelioid sarcoma & $3(1.9)$ & $2(2.8)$ & $1(1.2)$ & \\
\hline other soft tissue sarcoma & $3(1.9)$ & $2(2.8)$ & $1(1.2)$ & \\
\hline other osteogenic sarcoma & $2(1.3)$ & $1(1.4)$ & $1(1.2)$ & \\
\hline angiosarcoma & $2(1.3)$ & $2(2.8)$ & $0(0.0)$ & \\
\hline malignant peripheral nerve sheath tumor & $2(1.3)$ & $0(0.0)$ & $2(2.4)$ & \\
\hline undifferentiated sarcoma & $2(1.3)$ & $0(0.0)$ & $2(2.4)$ & \\
\hline chemotherapy regimens & & & & 0.082 \\
\hline$A D$ & $2(1.3)$ & $2(2.8)$ & $0(0.0)$ & \\
\hline $\mathrm{Al}$ & $72(46.2)$ & $28(38.9)$ & $44(52.4)$ & \\
\hline $\mathrm{CAV} / \mathrm{IE}$ & $12(7.7)$ & $5(6.9)$ & 7 (8.3) & \\
\hline GT & $25(16.0)$ & $9(12.5)$ & $16(19.0)$ & \\
\hline MAID & $21(13.5)$ & $14(19.4)$ & 7 (8.3) & \\
\hline $\mathrm{T} 10$ & $23(14.7)$ & $13(18.1)$ & $10(11.9)$ & \\
\hline TA & $1(0.6)$ & $1(1.4)$ & $0(0.0)$ & \\
\hline
\end{tabular}

N/A, Not Available. 


\section{Efficacy Evaluation}

Both short-term efficacy and long-term efficacy were evaluated. Short-term efficacy was mainly evaluated at 12 weeks, and the evaluation indexes included complete response (CR), partial response $(\mathrm{PR})$, stable disease $(\mathrm{SD})$, progressive disease $(\mathrm{PD})$, the objective response rate (ORR) and the disease control rate (DCR). Long-term efficacy was evaluated at the end of follow-up, and the indexes included CR, PR, SD, PD, the ORR, the DCR, the median progression-free survival (mPFS) and the mOS. The ORR was calculated as $(\mathrm{CR}+\mathrm{PR}) /$ total cases $\times 100 \%$. The DCR was calculated as $(\mathrm{CR}+\mathrm{PR}+\mathrm{SD}) /$ total cases $\times 100 \%$. PFS was defined as the time from the start of treatment to disease progression; OS was defined as the time from the start of treatment to death from any cause.

\section{Safety and Toxicity Assessments}

156 patients underwent efficacy and safety assessments. Treatment-related adverse events were assessed and graded based on the National Cancer Institute Common Terminology Criteria for Adverse Events (CTCAE, version 5.0).

\section{Statistical Analysis}

All data were analyzed using SPSS 22.0. Chi-square test was used to compare clinical pathologic features, ORR, DCR and adverse events of the two groups.

PFS and OS were calculated by the life table method. KaplanMeier and Cox analyses were used to compare PFS between the Endostar combined with chemotherapy and traditional chemotherapy groups, and a $\mathrm{p}$ value $<0.05$ was considered statistically significant.

\section{RESULTS}

\section{Patient Demographics}

All 156 patients (Figure 1B) had stage IV sarcoma according to the American Joint Committee on Cancer (AJCC) 8th edition staging system. According to the treatment methods, the patients were divided into two groups: 84 were included in the Endostar combined with chemotherapy group (combined group) (Figure 1C), and 72 were included in the traditional chemotherapy group (control group) (Figure 1D). In this study, there were 95 males and 61 females, with a median age of 45.50 years (range, 10-81 years). The most common pathological types were UPS $(n=38)$, synovial sarcoma $(n=29)$, osteosarcoma $(n=28)$, rhabdomyosarcoma $(n=13)$, and liposarcoma $(n=12)$, etc. (Figure 1B and Table 1). There was no significant difference in age, sex, location, pathological classification, chemotherapy regimens, Eastern Cooperative Oncology Group (ECOG) performance status, previous surgery type, previous radiotherapy or metastatic site between these two groups (all p>0.05) (Table $\mathbf{1}$ ).

\section{Combination Therapy Significantly Improved the ORR}

Target lesions were evaluated according to the RECIST 1.1. Changes in the maximum diameter of the target lesions in the
Endostar combined with chemotherapy group and traditional chemotherapy group (for optimal efficacy) were shown in Figures 2A, B. Also the changes in the target lesions in the two groups during treatment are shown in Figures 2C, D.

In terms of short-term efficacy, we found obvious differences between the two groups. At 12th week, all patients received at least two cycles of Endostar combined with chemotherapy or chemotherapy alone. Of the 84 patients in the combined group, 5 (5.9\%, 5/84) achieved CR, 29 (34.5\%, 29/84) achieved PR, 33 (39.3\%, 33/84) achieved SD and 17 (20.2\%, 17/84) achieved PD. The 12 -week ORR was $40.5 \%$ (34/84), and the DCR was $79.8 \%$ $(67 / 84)$. Among the 72 patients in the chemotherapy group, no patients achieved CR (0\%, 0/72), $13(18.1 \%, 13 / 72)$ achieved PR, $40(55.6 \%, 40 / 72)$ achieved SD, and $19(26.4 \%, 19 / 72)$ achieved PD. The 12 -week ORR was $18.1 \%(13 / 72)$, and the DCR was $73.6 \%(53 / 72)$ (Table 2). The ORR in the combined group at week 12 was significantly higher than that in the chemotherapy group $(\mathrm{p}=0.002)$.

Given the fact that chemotherapy and targeted therapy would have secondary resistance and disease progression after multiple cycles, we especially paid attention to the best ORR/DCR and the final ORR/DCR. During the following observation, we noticed that there were some patients who suffered from disease progression both groups, suggesting that the ORR/DCR in the 12th week might be the best ORR/DCR in the both groups in this study.

\section{Combination Therapy Significantly Improved the MPFS of Stage IV Soft Tissue Sarcoma Patients}

The median follow-up period was 16.9 months, ranged from 2.5 months to 118.1 months. By the end of follow-up, the ORR was $10.7 \%$, the DCR was $26.2 \%$, the mPFS reached 10.42 months, and the mOS was 26.84 months in the combined group. In the chemotherapy group, the ORR was $1.4 \%$, the DCR was $5.6 \%$, the mPFS was 6.87 months, and the mOS was 23.56 months. The ORR and DCR of the combined group were significantly higher than those of the chemotherapy group (Table 2). Most importantly, there was a significant difference in the mPFS between the combined group and chemotherapy group (10.42 months VS 6.87 moths; $\mathrm{p}=0.003$ ) (Figure 3A). However, there was no significant difference in the $\operatorname{mOS}(\mathrm{p}=0.481)$ (Figure 3B).

We analyzed the clinical and pathological features affecting the mPFS. In the univariate Cox analysis, only the pathological classification, location, and combined Endostar to chemotherapy were associated with the mPFS (Figure 4). However, in the multivariate Cox analysis, only the pathological sarcoma subtypes (soft tissue sarcoma vs osteogenic sarcoma, $\mathrm{p}<0.001$, HR: 0.383, 95\% CI: 0.251-0585) and therapy regiment (Endostar plus chemotherapy vs chemotherapy, $\mathrm{p}=0.033$, HR: $0.675,95 \%$ CI: $0.470-0.969)$ were associated with the improved mPFS.

We then tried to find out which sarcoma subtype could benefit from the combined therapy with improved mPFS. Our study included 36 patients with osteogenic sarcomas and 120 patients with soft tissue sarcomas. In the osteogenic sarcoma group, the mPFS of the combined group and chemotherapy 
A

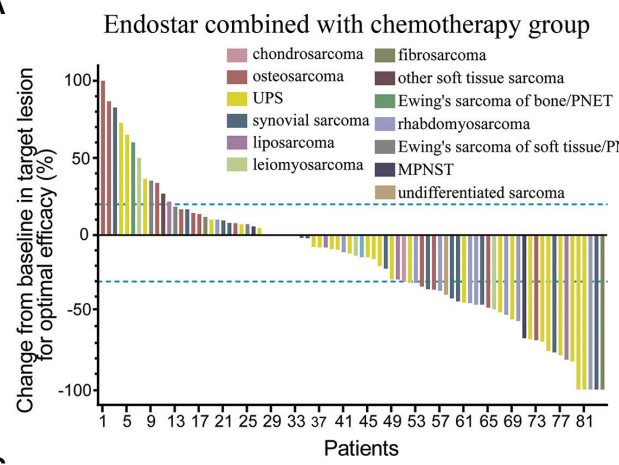

C

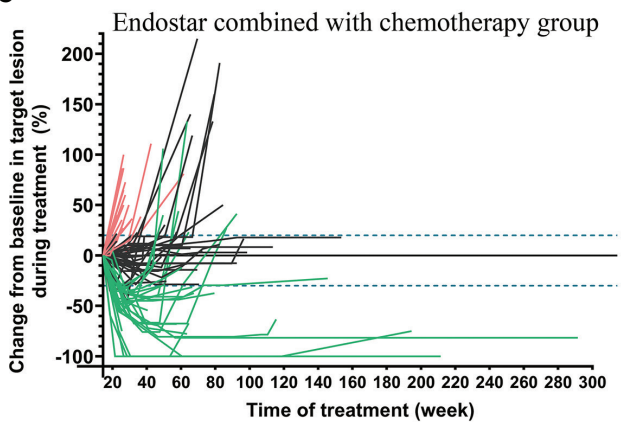

B
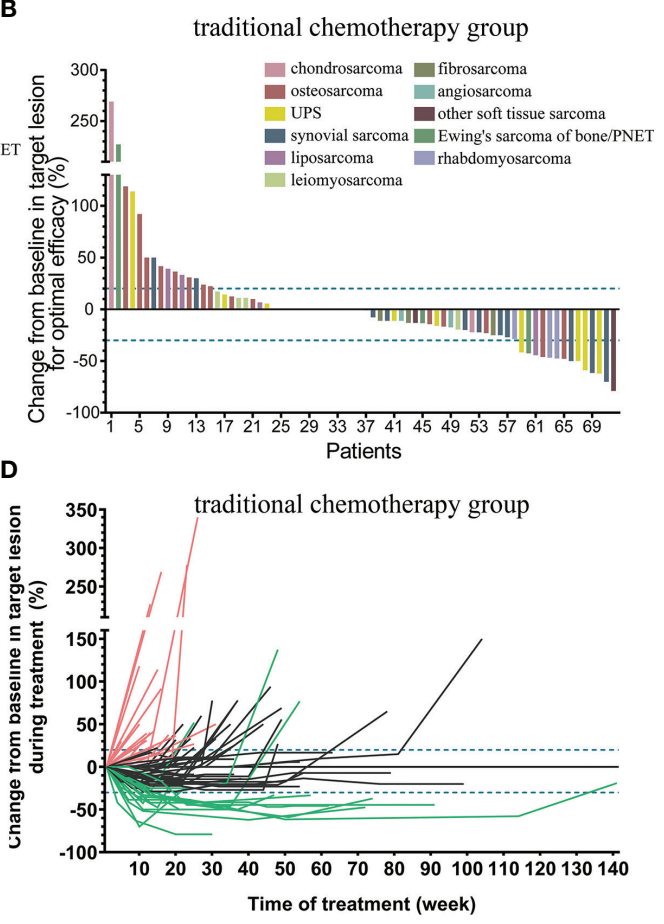

FIGURE 2 | Changes in the maximum diameter of the target lesion in the Endostar combined with chemotherapy group (A) and traditional chemotherapy group (B) (for optimal efficacy). Continuous changes in the target lesions in the Endostar combined with chemotherapy group (C) and traditional chemotherapy group (D) during treatment.

group were 3.22 months vs. 3.78 months $(\mathrm{p}=0.59)$, and the mOS were 10.94 months vs. 16.85 months $(\mathrm{p}=0.55)$, respectively, showing no significant improvement (Figures 3C, D). For the soft tissue sarcoma patients, the mPFS of the combined therapy group was significantly improved (11.27 months vs 8.05 months, $\mathrm{p}=0.004)$, even the mOS (26.88 months vs 26.81 months, $\mathrm{p}=0.536$ ) showed no difference between these two groups (Figures 3E, F). It suggested that the soft tissue sarcomas could significantly benefit from the combined therapy and achieve better mPFS.

TABLE 2 | The efficacy in chemotherapy and endostar combined with chemotherapy.

Efficacy at the 12th week

\begin{tabular}{|c|c|c|c|}
\hline & Chemotherapy & endostar combined with chemotherapy & $\mathrm{p}$ value \\
\hline $\mathrm{CR}$ & $0 / 72,0 \%$ & $5 / 84,5.9 \%$ & NA \\
\hline PD & $19 / 72,26.4 \%$ & $17 / 84,20.2 \%$ & 0.363 \\
\hline ORR & $18.1 \%(13 / 72)$ & $40.5 \%(34 / 84)$ & 0.002 \\
\hline DCR & $73.6 \%(53 / 72)$ & $79.8 \%(67 / 84)$ & 0.363 \\
\hline Patients number & 72 & 84 & \\
\hline $\mathrm{CR}$ & $0 / 72,0 \%$ & $4 / 84,4.8 \%$ & NA \\
\hline PR & $1 / 72,1.4 \%$ & $5 / 84,5.9 \%$ & 0.289 \\
\hline SD & $3 / 72,4.2 \%$ & $13 / 84,15.5 \%$ & 0.02 \\
\hline PD & $68 / 72,94.4 \%$ & $62 / 84,73.8 \%$ & 0.001 \\
\hline ORR & $1.4 \%(1 / 72)$ & $10.7 \%(9 / 84)$ & 0.041 \\
\hline
\end{tabular}

N/A, Not Available. 
A

mPFS in entire cohort

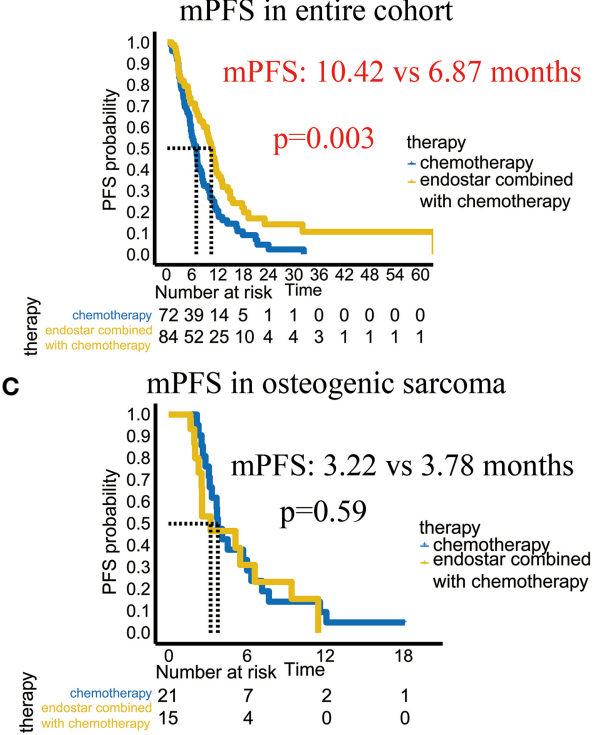

E

mPFS in STS

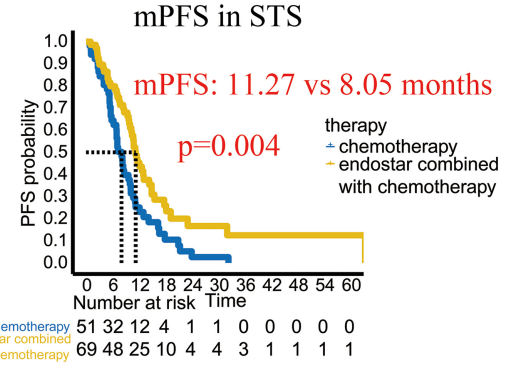

G mPFS in UPS

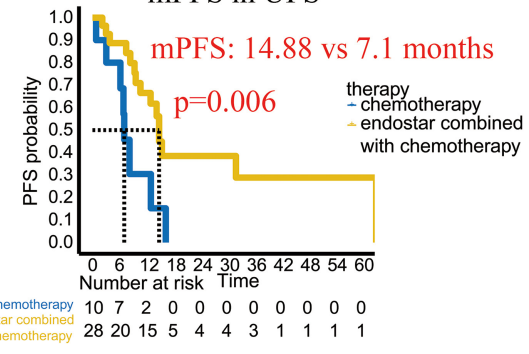

I

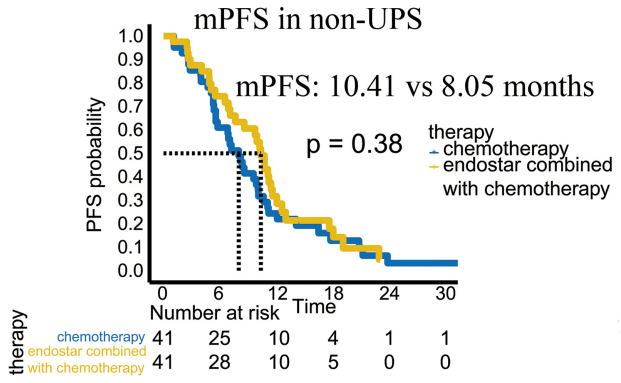

B

mOS in entire cohort

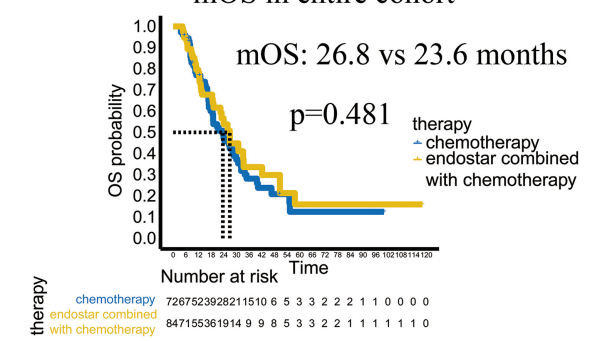

D mOS in osteogenic sarcoma
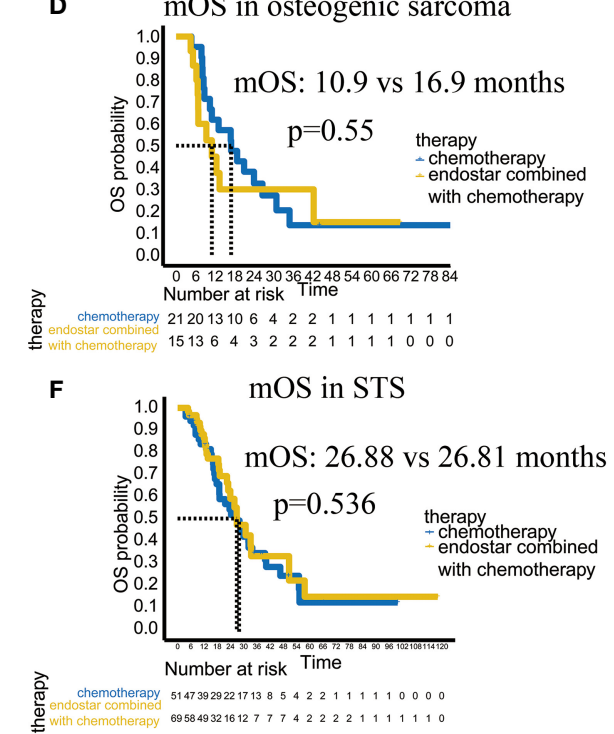

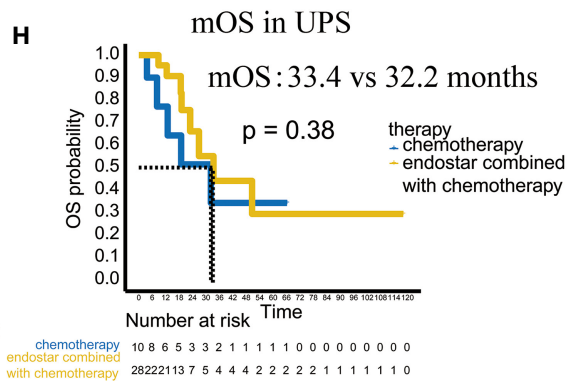

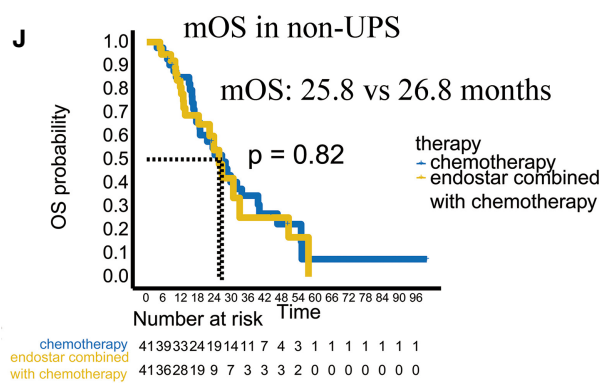

FIGURE 3 | Comparison of the MPFS and mOS between the Endostar combined chemotherapy group and chemotherapy group. (A) Comparison of the mPFS in the entire cohort. (B) Comparison of the mOS in the entire cohort. (C) Comparison of the mPFS in patients with osteogenic sarcoma. (D) Comparison of the mOS in patients with osteogenic sarcoma. (E) Comparison of the mPFS in patients with soft tissue sarcoma. (F) Comparison of the mOS in patients with soft tissue sarcoma. (G) Comparison of the mPFS in patients with UPS. (H) Comparison of the mOS in patients with UPS. (I) Comparison of the mPFS in patients with nonUPS. (J) Comparison of the mOS in patients with non-UPS. 


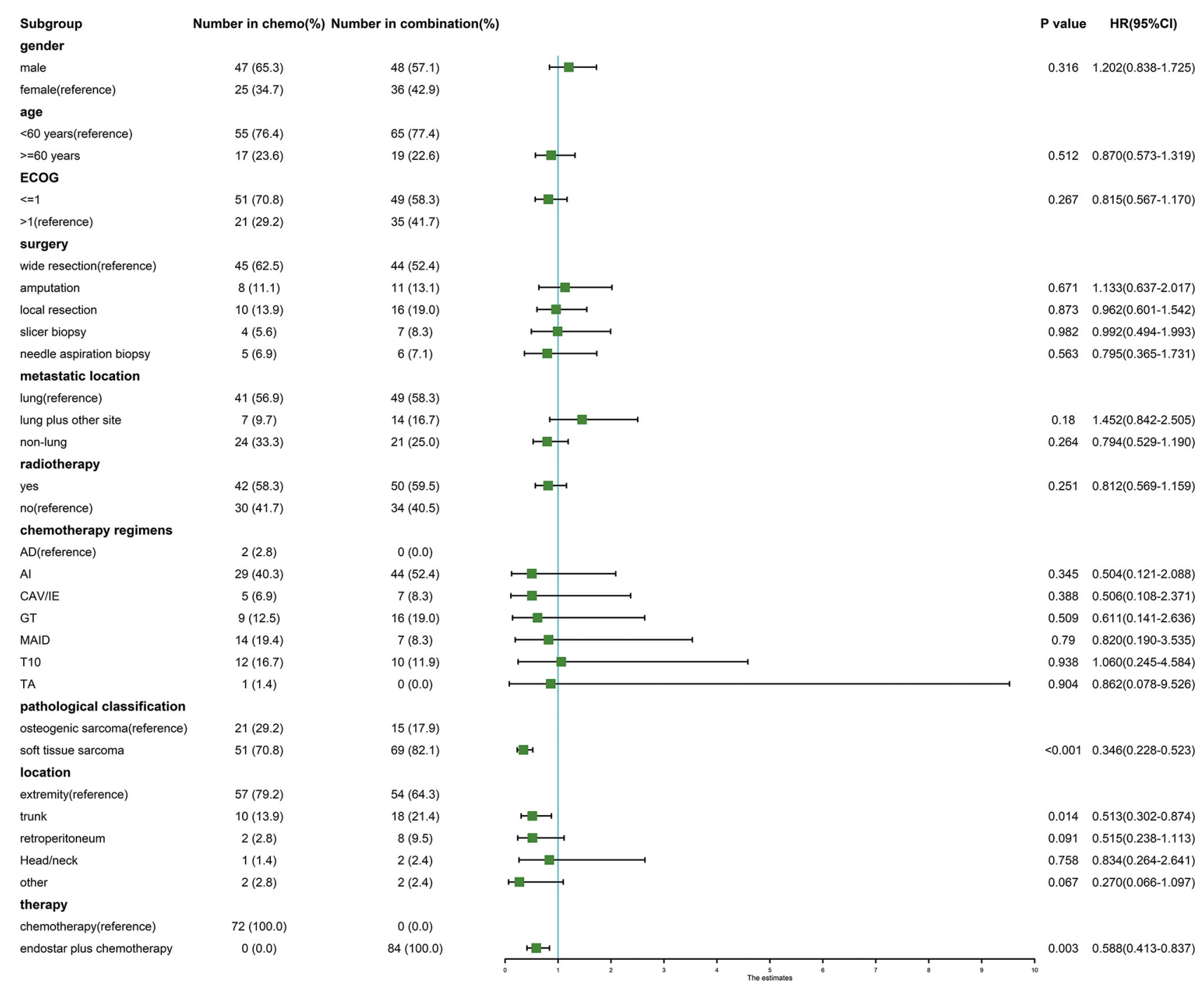

FIGURE 4 | Forest plot of clinicopathological features affecting the mPFS in the univariate Cox analysis.

\section{UPS Patients Significantly Benefited From Endostar Combined With Chemotherapy With Improved PFS}

Due to the fact that it is the soft tissue sarcoma patients which were significantly benefited from the combined therapy, we further analyzed which soft tissue sarcoma subtype could benefit from the combined therapy. The results revealed that the patients with UPS achieved the better efficacy of Endostar combined with chemotherapy. In this study, there were 38 patients with UPS: 28 patients in the combined group and 10 patients in the chemotherapy group. In the Cox analysis, the chemotherapy regimen and location did not affect the mPFS, but the addition of Endostar to chemotherapy did affect the mPFS ( $p=0.01$ ). The mPFS of the combined group was up to 14.88 months, while it was only 7.1 months in the chemotherapy group, with a significant difference $(p=0.006)$ (Figure 3G). However, there was no statistically significant difference in the mOS $(\mathrm{p}=0.38)$ (Figures 3G, H). Among the remaining soft tissue sarcoma subtypes, there was no significant difference in the mPFS $(\mathrm{p}=0.38)$ or $\operatorname{mOS}(\mathrm{p}=0.82)$ between the combined group and the chemotherapy group (Figures 3I, J). It suggested that the UPS patients treated with combined therapy could achieve better efficacy with improved mPFS.

Additionally, 4 patients suffered from PD after receiving traditional chemotherapy then transfered to the Endostar combined with chemotherapy treatments. But the number of patients was too small to compare whether there was a difference in the mPFS. The first patient developed lung metastases after receiving traditional chemotherapy and was then treated with Endostar combined with AI. After two cycles of combination treatment, PD was achieved, the final PFS was 2.3 months, and the OS was 10.94 months. The second patient developed lung 
metastasis after three cycles of AI chemotherapy and then received Endostar combined with GT. The efficacy was evaluated as SD at 12 weeks and PD at the end of follow-up, with a final PFS duration of 19.22 months and an OS duration of 23.92 months. The third patient progressed after receiving MAID and GT chemotherapy, followed by Endostar combined with AI. After 6 cycles of combination treatment, the efficacy was evaluated as PR at 12 weeks and PD at the end of follow-up, with a final PFS of 23.06 months and an OS of 30.55 months. The fourth patient progressed after one cycle of treatment with ifosfamide plus liposomal paclitaxel and progressed again after two cycles of treatment with ifosfamide plus liposomal doxorubicin and Endostar, with a final PFS of 2.5 months and an OS of 4.7 months.

\section{Combination Therapy Might Increase Chemotherapy Sensitivity and Help Achieve CR in Some Soft Tissue Sarcoma Patients}

No patient achieved CR in the chemotherapy group, but four patients achieved $\mathrm{CR}$ in the Endostar combined with chemotherapy group. Figures $\mathbf{5 A - F}$ shows the PET-CT comparison of a typical patient who achieved CR before and after treatment with Endostar combined with AI. This was a UPS patient with a large retroperitoneal tumor. Pretreatment imaging showed a large retroperitoneal mass, approximately $13.5 \mathrm{~cm}$ in diameter, with significant invasion into the lumbar spine (Figures 5A-C). The patient was continuous in pain and could not lie on his back. After treatment with 4 cycles of Endostar combined with AI, the retroperitoneal tumor and lumbar spine lesion disappeared, PETCT suggested no concentration aggregation, and the efficacy was evaluated as CR (Figures 5D-F). Figure 6 shows another typical patient who achieved CR before and after treatment with Endostar combined with chemotherapy. This was a patient who developed pulmonary metastases after surgery of the thigh synovial sarcoma. After 6 cycles of Endostar combined with AI, the target lesion in the right lung had completely disappeared on CT (Figures 6A-H).

In addition to the four patients achieved CR, there were five patients who achieved PR in the Endostar combined with chemotherapy group. MPNST is considered a typical chemotherapy-resistant sarcoma type. The combination of chemotherapy with Endostar significantly increased chemotherapy sensitivity. A patient with MPNST in the right thigh had metastases in both lungs detected at the initial consultation. After giving the patient 4 cycles of Endostar combined with AI, imaging showed a significant reduction in both the primary lesion and lung metastases in the right thigh (Figures 7A-F). The patient was treated with palliative surgery and then received maintenance therapy.

\section{Endostar Combined With Chemotherapy Did Not Increase the Frequency or Severity of Adverse Events Compared With Traditional Chemotherapy}

In this study, the majority of adverse events in the combined group were mild (grades 1-2) and manageable. Specifically, grade
1 adverse events accounted for 80\% (177/221), grade 2 adverse events accounted for $14 \%(31 / 221)$, grade 3 adverse events accounted for $2.7 \%(6 / 221)$, and grade 4 adverse events accounted for $3.2 \%$ (7/221). Myelosuppression was the most common adverse event in the combined group (25.8\%, 57/221). Of the patients in this group, seven developed grade IV myelosuppression, and six recovered completely after treatment and continued to receive Endostar combined with chemotherapy; however, the remaining patient discontinued treatment due to adverse events. Other common adverse events included gastrointestinal reactions (24.9\%, 55/221), abnormal liver function $(23.1 \%, 51 / 221)$, pigmentation $(9.5 \%$, $21 / 221)$, arrhythmia $(9 \%, 20 / 221)$, allergies $(5 \%, 11 / 221)$, and renal inadequacy $(2.7 \%, 6 / 221)$ (Table 3 ).

Next, we compared the incidence of adverse events between the Endostar combined with chemotherapy group and the traditional chemotherapy group. The results showed no statistically significant difference in the incidence of various adverse events between the two groups (Table 4). Furthermore, we compared the incidence of various adverse events between UPS patients and non-UPS patients in soft tissue sarcoma patients who received Endostar combined with chemotherapy. The results showed no significant difference in the incidence of various adverse events between the two groups of patients (Table 5). These results suggest that Endostar combined with chemotherapy does not cause additional adverse events compared to chemotherapy alone, and there is no significant difference in the incidence of adverse events for UPS patients.

\section{DISCUSSION}

Chemotherapy plays an indispensable role in the treatment of stage IV bone and soft tissue sarcoma. Although improvements in surgical procedures have allowed most patients to retain extremity function, overall survival in patients with sarcoma has not improved significantly in recent years (11). Therefore, it is urgent to find a therapeutic method that can effectively improve the chemotherapy sensitivity of patients with tolerable toxicity. In this background, people ushered in the era of antiangiogenic therapy. Endostar, a synthetic recombinant endostatin, has a broad spectrum of antiangiogenic activities mainly by targeting VEGF (22). As early as 2005, Endostar was approved by the China Food and Drug Administration for the treatment of non-small cell lung cancer (34). However, few studies have been conducted in bone and soft tissue sarcoma. Our previous study indicated that Endostar combined with chemotherapy could improve PFS in patients with advanced sarcoma (33). In the present study, we found that the pathological classification and combination treatment were independent risk factors affecting sarcoma patient outcomes. Endostar combined with chemotherapy significantly improved the mPFS of the soft tissue sarcoma patients, especially the UPS. In addition, Endostar combined with chemotherapy did not increase the incidence or severity of adverse events compared 
A

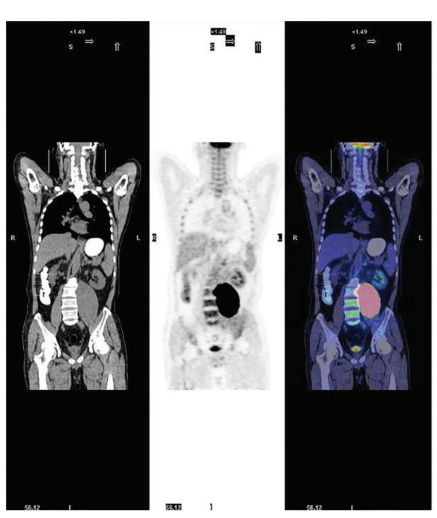

B
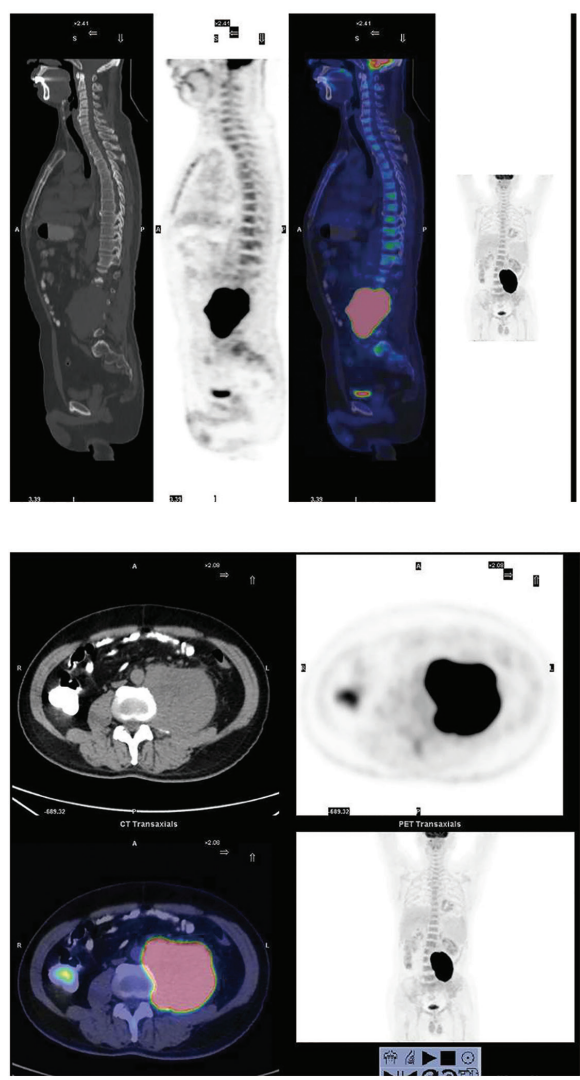

$2016 / 10 / 07$
D

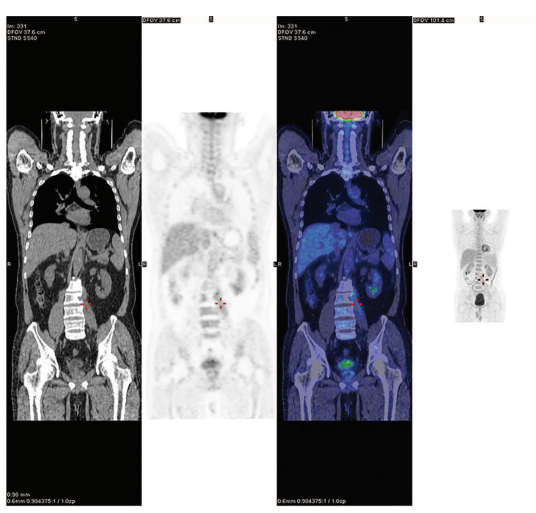

E

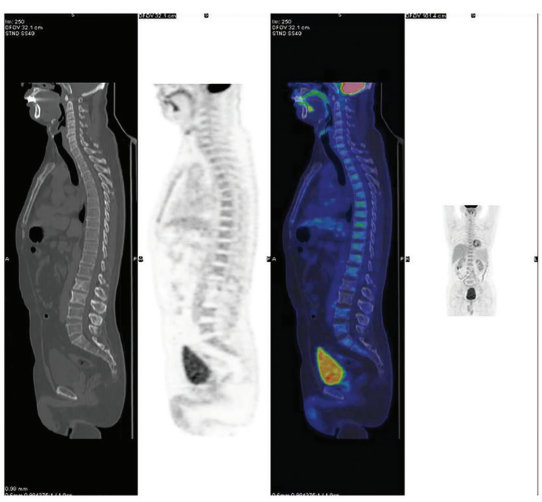

$\mathbf{F}$

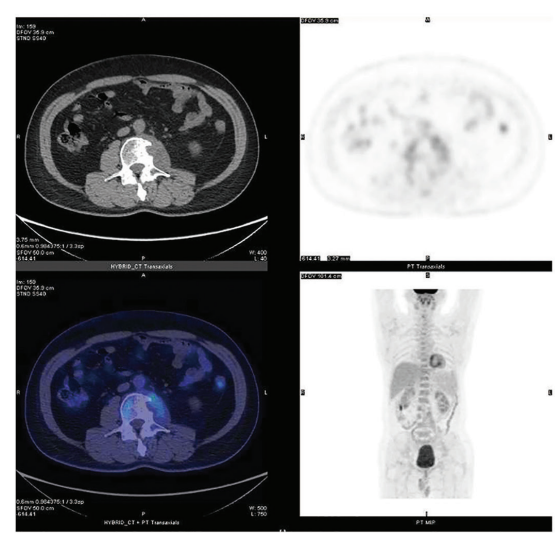

$2017 / 2 / 13$

FIGURE 5 | PET-CT comparison of a typical patient with a large retroperitoneal UPS who achieved CR before and after treatment with Endostar combined with Al. (A) Coronal PET-CT image before treatment. (B) Sagittal PET-CT image before treatment. (C) Cross-sectional PET-CT image before treatment. (D) Coronal PET-CT image after treatment. (E) Sagittal PET-CT image after treatment. (F) Cross-sectional PET-CT image after treatment.

to traditional chemotherapy, and the adverse events were tolerable and well controlled.

We believe that this study has the following two advantages. First, this is the largest cohort retrospective study on the efficacy of Endostar combined with chemotherapy in bone and soft tissue sarcoma, with 156 patients enrolled. Due to the rarity of bone and soft tissue sarcoma, few studies to date have evaluated the efficacy and safety of Endostar combined with chemotherapy in bone and soft tissue sarcoma, and most studies have included only a few dozen patients $(11,31-33)$. One study included 116 patients, but only patients with osteosarcoma were enrolled, and patients with soft tissue sarcoma and other osteogenic sarcoma and soft tissue 
A

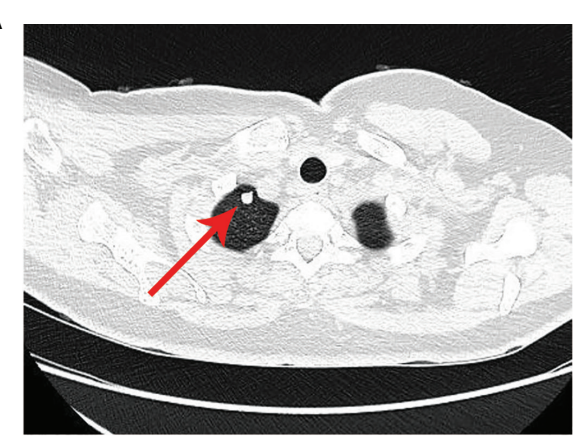

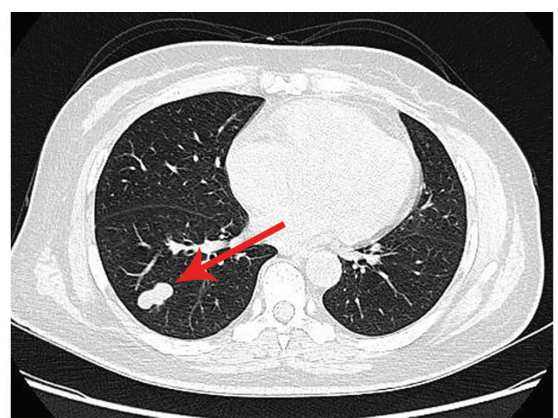

2019-03-07

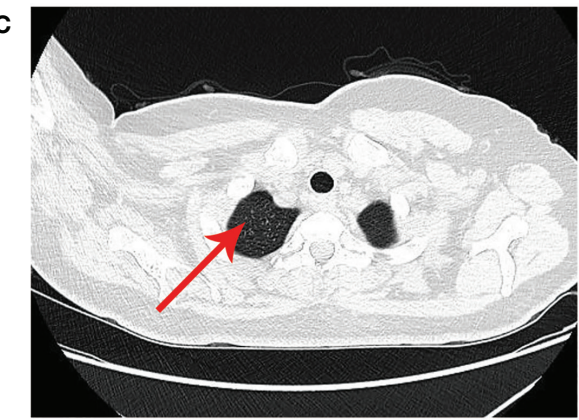

D

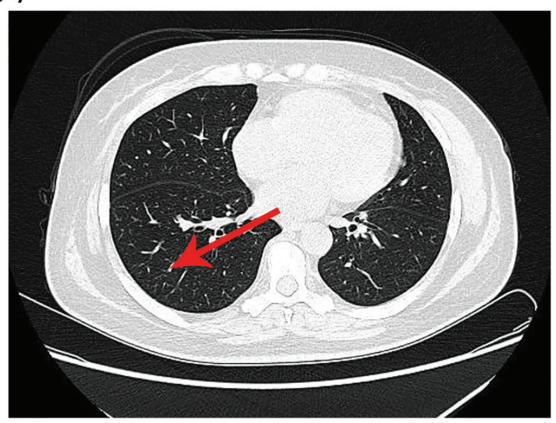

2019-06-06

E

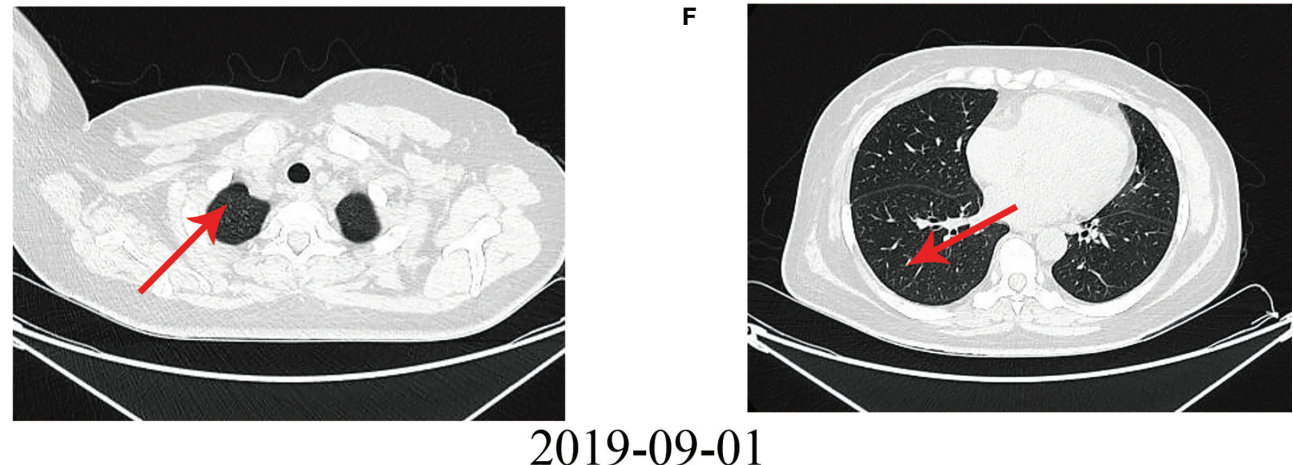

G

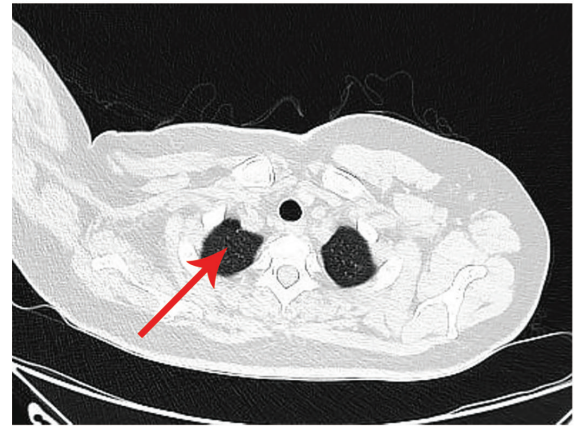

2019-11-25

FIGURE 6 | Another typical patient with pulmonary metastases who achieved CR after surgery for thigh synovial sarcoma before and after treatment with Endostar combined with chemotherapy. (A) Upper lobe metastases before treatment. (B) Lower lobe metastases before treatment. (C-H) Metastases in the upper and lower lobes disappeared after treatment with Endostar combined with chemotherapy. 
A

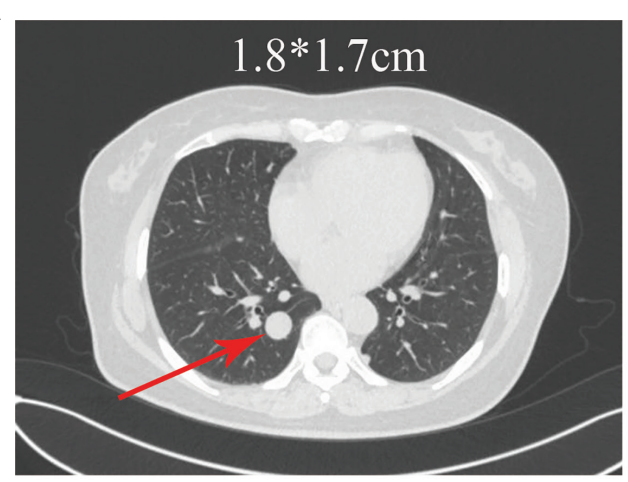

B

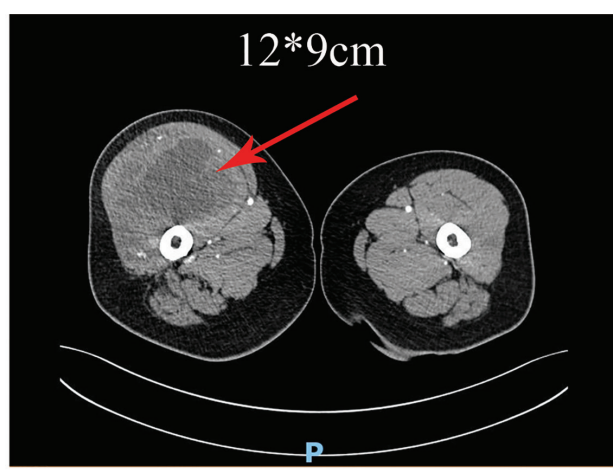

2019-03-07

C

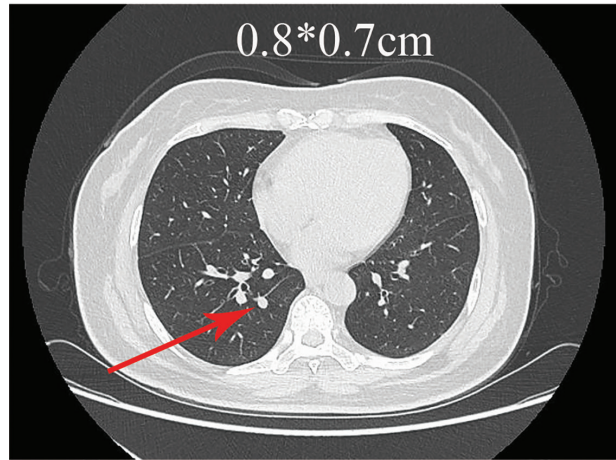

D

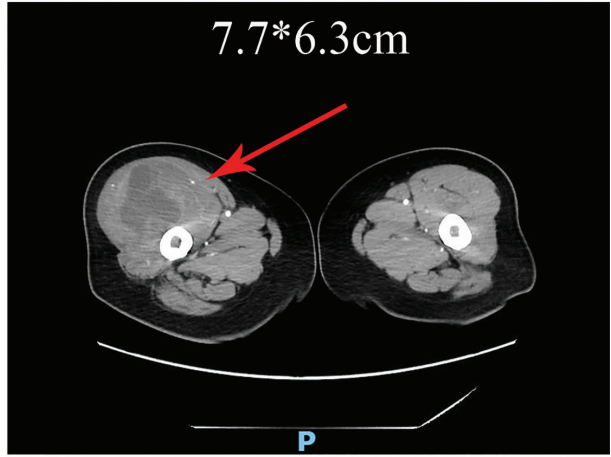

2019-05-15

$\mathbf{E}$

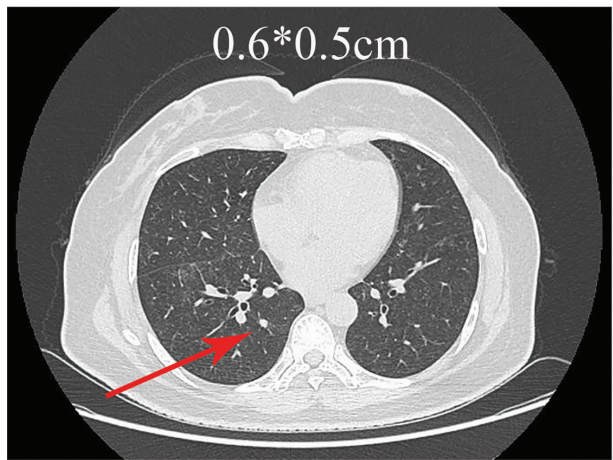

$\mathbf{F}$

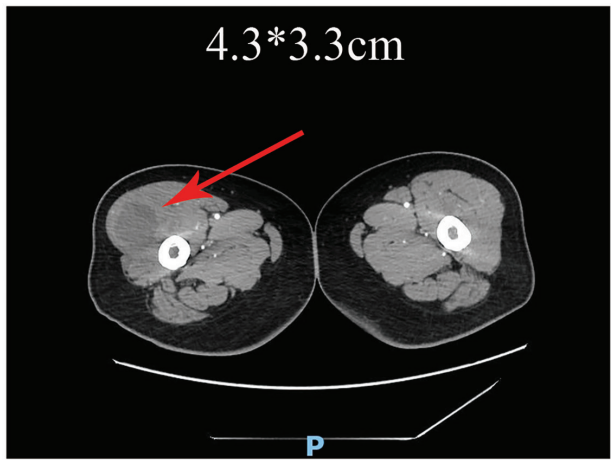

2019-07-25

FIGURE 7 | A typical patient with a malignant peripheral nerve sheath tumor in the right thigh and metastases in both lungs detected at the initial consultation who achieved PR. (A) Lung metastases before treatment. (B) Primary tumor of the right thigh before treatment. (C-F) After receiving Endostar combined with chemotherapy, both the lung metastases and the primary tumor of the right thigh were reduced.

sarcoma were excluded (11). The small sample size weakened the credibility of these findings to some extent. However, the present study overcame this shortcoming and enrolled 156 patients, which enhanced the credibility of the results. The results showed that Endostar combined with chemotherapy improved patient prognosis at 12th week and at the end of follow-up. At the end of follow-up, Endostar combined with chemotherapy significantly improved the mPFS (10.42 months vs. 6.87 months, $\mathrm{p}=0.003$ ). These findings are similar to those of two previously reported retrospective studies $(11,32)$. These results confirmed that Endostar combined with chemotherapy can indeed improve the prognosis of patients. Second, we screened the most sensitive pathological subtypes to Endostar combined with chemotherapy for the first time. Sarcoma is a very heterogeneous malignant 
TABLE 3 | The adverse events in the endostar combined with chemotherapy.

\begin{tabular}{|c|c|c|c|c|c|}
\hline Adverse Event & Grade1 & Grade 2 & Grade 3 & Grade 4 & Total \\
\hline myelosuppression & 35 & 11 & 4 & 7 & 57 (57/221, 25.8\%) \\
\hline gastrointestinal reactions & 53 & 1 & 1 & 0 & 55 (55/221, 24.9\%) \\
\hline allergies & 9 & 1 & 1 & 0 & $11(11 / 221,5 \%)$ \\
\hline pigmentation & 14 & 7 & 0 & 0 & $21(21 / 221,9.5 \%)$ \\
\hline abnormal liver function & 46 & 5 & 0 & 0 & $51(51 / 221,23.1 \%)$ \\
\hline arrhythmia & 17 & 3 & 0 & 0 & $20(20 / 221,9 \%)$ \\
\hline renal inadequacy & 3 & 3 & 0 & 0 & $6(6 / 221,2.7 \%)$ \\
\hline Total & 177 & 31 & 6 & 7 & 221 \\
\hline
\end{tabular}

tumor with more than 50 pathological subtypes. Whether all pathological subtypes or only specific pathological subtypes are sensitive to Endostar combined with chemotherapy remains unknown. In the present study, the pathological classification and addition of Endostar to chemotherapy were identified as independent risk factors affecting the mPFS. To control for the influence of the pathological classification on the mPFS, we compared the effect of the addition of Endostar to chemotherapy on the mPFS according to different pathological classifications. In osteogenic sarcoma, the presence or absence of Endostar had no effect on the mPFS ( $p=0.59$ ), while in soft tissue sarcoma, the mPFS of patients treated with Endostar combined with chemotherapy was significantly higher than that of patients treated with traditional chemotherapy $(p=0.004)$. Considering that 12 pathological classifications of soft tissue sarcoma were included in this study, we further screened the pathological classifications. The results showed that the mPFS was significantly higher in UPS patients receiving Endostar combined with chemotherapy $(\mathrm{p}=0.006)$. These results suggest that UPS might be the possible pathological subtype that benefits from Endostar combined with chemotherapy. Therefore, Endostar combined with chemotherapy can be applied to improve the prognosis of patients with UPS in future treatment.

In contrast to hand-foot syndrome, hypertension, proteinuria and other common adverse events of antiangiogenic drugs, such as bevacizumab and apatinib, the main adverse events of Endostar were cardiotoxicity, gastrointestinal reactions and allergic reactions (34-38). Adverse events of chemotherapy mainly include myelosuppression, abnormal liver function, gastrointestinal reactions and so on $(5,39)$. In this study, the major adverse events of Endostar combined with chemotherapy were myelosuppression $(25.8 \%)$, gastrointestinal reactions $(24.9 \%)$ and abnormal liver function (23.1\%), and the majority of adverse events were grades I and II. Moreover, in terms of the incidence of adverse events, the incidence in the Endostar combined with chemotherapy group was not higher than that in the traditional chemotherapy group. Furthermore, in terms of the only pathological subtype of soft tissue sarcoma that can benefit from endostar combined with chemotherapy, the incidence of various adverse events in UPS patients receiving Endostar combined with chemotherapy was not higher than that in non-UPS patients. Therefore, Endostar combined with chemotherapy did not increase the incidence of adverse events, and these adverse events were controllable and tolerated, indicating that Endostar combined with chemotherapy not only improves the prognosis of patients but is also safe.

Nevertheless, this study still has several drawbacks. First, this was a single-center retrospective study that needs to be validated through a multicenter prospective clinical trial. Second, although the chemotherapy regimen did not affect the mPFS in this study, there were many types of chemotherapy regimens that were not randomly selected, and a randomized, double-blind clinical trial is needed for further validation. Third, in this study, although the number of UPS patients was 38, the number of patients with other pathological types was still relatively small, and the inclusion of other pathological types is needed to further evaluate the efficacy and safety of Endostar combined with chemotherapy in patients with advanced sarcoma. Fourth, previous studies reported that Endostar combined with chemotherapy could improve the mPFS in patients with osteosarcoma (11). However, the results of the present study showed that Endostar combined with chemotherapy could not improve the prognosis of patients with osteosarcoma. The next step is to increase the number of patients with osteogenic sarcoma, including those with osteosarcoma, to further evaluate the efficacy of Endostar combined with chemotherapy.

In conclusion, Endostar combined with chemotherapy significantly improved the mPFS in patients with advanced soft

TABLE 4 | Comparison of adverse events between endostar combined with chemotherapy and chemotherapy.

myelosuppression
gastrointestinal reactions
abnormal liver function
pigmentation
arrhythmia
allergies
renal inadequacy

\begin{tabular}{c}
\hline control group \\
$54(54 / 72,75 \%)$ \\
$54(54 / 72,75 \%)$ \\
$44(44 / 72,61.1 \%)$ \\
$10(10 / 72,13.9 \%)$ \\
$13(13 / 72,18.1 \%)$ \\
$11(11 / 72,15.3 \%)$ \\
$3(3 / 72,4.2 \%)$
\end{tabular}

combined group

$\begin{array}{cc}57(57 / 84,67.9 \%) & 0.326 \\ 55(55 / 84,65.5 \%) & 0.196 \\ 51(51 / 84,60.7 \%) & 0.960 \\ 21(21 / 84,25 \%) & 0.083 \\ 20(20 / 84,23.8 \%) & 0.380 \\ 11(11 / 84,13.1 \%) & 0.696 \\ 6(6 / 84,7.1 \%) & 0.652\end{array}$


TABLE 5 | Comparison of adverse events between UPS and non-UPS in combined group.

\begin{tabular}{|c|c|c|c|}
\hline \multirow[t]{2}{*}{ Adverse Event } & \multicolumn{2}{|c|}{ No. (\%) Adverse Events by Treatment } & \multirow[t]{2}{*}{$\boldsymbol{P}$} \\
\hline & UPS & non-UPS & \\
\hline myelosuppression & $20(20 / 28,71.4 \%)$ & 37 (37/56, 66.1\%) & 0.620 \\
\hline gastrointestinal reactions & 19 (19/28, 67.9\%) & $36(36 / 56,64.3 \%)$ & 0.746 \\
\hline abnormal liver function & $18(18 / 28,64.3 \%)$ & $33(33 / 56,58.9 \%)$ & 0.636 \\
\hline pigmentation & $5(5 / 28,17.9 \%)$ & $16(16 / 56,28.6 \%)$ & 0.285 \\
\hline arrhythmia & $6(6 / 28,21.4 \%)$ & $14(14 / 56,25.0 \%)$ & 0.717 \\
\hline allergies & $3(3 / 28,10.7 \%)$ & $8(8 / 56,14.3 \%)$ & 0.909 \\
\hline renal inadequacy & $1(1 / 28,3.6 \%)$ & $5(5 / 56,8.9 \%)$ & 0.653 \\
\hline
\end{tabular}

tissue sarcoma, especially the UPS patients, and the adverse events were tolerable. This treatment regimen has shown encouraging objective efficacy and controllable toxicity. Therefore, Endostar combined with chemotherapy can be applied to improve the prognosis of patients with advanced soft tissue sarcoma. In the meantime, more patients need to be recruited, or more rigorous randomized controlled trials need to be conducted to further confirm these findings.

\section{DATA AVAILABILITY STATEMENT}

The raw data supporting the conclusions of this article will be made available by the authors, without undue reservation.

\section{ETHICS STATEMENT}

The studies involving human participants were reviewed and approved by Tianjin Medical University Cancer Institute and

\section{REFERENCES}

1. Trautmann F, Schuler M, Schmitt J. Burden of Soft-Tissue and Bone Sarcoma in Routine Care: Estimation of Incidence, Prevalence and Survival for Health Services Research. Cancer Epidemiol (2015) 39(3):440-6. doi: 10.1016/ j.canep.2015.03.002

2. Fujiki M, Miyamoto S, Kobayashi E, Sakuraba M, Chuman H. Early Detection of Local Recurrence After Soft Tissue Sarcoma Resection and Flap Reconstruction. Int Orthop (2016) 40(9):1975-80. doi: 10.1007/s00264-0163219-y

3. Yang Z, Zheng R, Zhang S, Zeng H, Li H, Chen W. Incidence, Distribution of Histological Subtypes and Primary Sites of Soft Tissue Sarcoma in China. Cancer Biol Med (2019) 16(3):565-74. doi: 10.20892/j.issn.20953941.2019.0041

4. Seddon B, Strauss SJ, Whelan J, Leahy M, Woll PJ, Cowie F, et al. Gemcitabine and Docetaxel Versus Doxorubicin as First-Line Treatment in Previously Untreated Advanced Unresectable or Metastatic Soft-Tissue Sarcomas (GeDDiS): A Randomised Controlled Phase 3 Trial. Lancet Oncol (2017) 18 (10):1397-410. doi: 10.1016/s1470-2045(17)30622-8

5. Lamplot JD, Denduluri S, Qin J, Li R, Liu X, Zhang H, et al. The Current and Future Therapies for Human Osteosarcoma. Curr Cancer Ther Rev (2013) 9 (1):55-77. doi: 10.2174/1573394711309010006

6. Marchandet L, Lallier M, Charrier C, Baud'huin M, Ory B, Lamoureux F. Mechanisms of Resistance to Conventional Therapies for Osteosarcoma. Cancers (Basel) (2021) 13(4):683. doi: 10.3390/cancers 13040683
Hospital Ethics Committee. The patients/participants provided their written informed consent to participate in this study.

\section{AUTHOR CONTRIBUTIONS}

ZL and CZ drafted and revised the manuscript. CZ and JY sent up the study and corrected the manuscript. ZL, TY, HL, CZ, SY, TL, RX, ST, YY, JZ, XB, and LZ performed experiments and analyzed data. GZ confirmed the pathological diagnosis. JY designed the project. All authors were actively involved in the preparation of this manuscript. All authors contributed to the article and approved the submitted version.

\section{FUNDING}

This work was partly supported by the Nature Science Foundation of Tianjin (grant number 16JCYBJC24100 to JY and 18YFZCSY00550 to JY).

7. Young RJ, Natukunda A, Litière S, Woll PJ, Wardelmann E, van der Graaf WTA. First-Line Anthracycline-Based Chemotherapy for Angiosarcoma and Other Soft Tissue Sarcoma Subtypes: Pooled Analysis of Eleven European Organisation for Research and Treatment of Cancer Soft Tissue and Bone Sarcoma Group Trials. Eur J Cancer (2014) 50(18):3178-86. doi: 10.1016/j.ejca.2014.10.004

8. Savina M, Le Cesne A, Blay JY, Ray-Coquard I, Mir O, Toulmonde M, et al. Patterns of Care and Outcomes of Patients With METAstatic Soft Tissue SARComa in a Real-Life Setting: The METASARC Observational Study. BMC Med (2017) 15(1):78. doi: 10.1186/s12916-017-0831-7

9. Liu J, Fan Z, Bai C, Li S, Xue R, Gao T, et al. Real-World Experience With Pembrolizumab in Patients With Advanced Soft Tissue Sarcoma. Ann Transl Med (2021) 9(4):339. doi: 10.21037/atm-21-49

10. Zucali PA, Bertuzzi A, Parra HJ, Campagnoli E, Quagliuolo V, Santoro A. The "Old Drug" Dacarbazine as a Second/Third Line Chemotherapy in Advanced Soft Tissue Sarcomas. Invest New Drugs (2008) 26(2):175-81. doi: 10.1007/ s10637-007-9086-z

11. Xu M, Xu CX, Bi WZ, Song ZG, Jia JP, Chai W, et al. Effects of Endostar Combined Multidrug Chemotherapy in Osteosarcoma. Bone (2013) 57 (1):111-5. doi: 10.1016/j.bone.2013.07.035

12. Tap WD, Jones RL, Van Tine BA, Chmielowski B, Elias AD, Adkins D, et al. Olaratumab and Doxorubicin Versus Doxorubicin Alone for Treatment of Soft-Tissue Sarcoma: An Open-Label Phase 1b and Randomised Phase 2 Trial. Lancet (2016) 388(10043):488-97. doi: 10.1016/s0140-6736(16)30587-6

13. Tap WD, Wagner AJ, Schoffski P, Martin-Broto J, Krarup-Hansen A, Ganjoo $\mathrm{KN}$, et al. Effect of Doxorubicin Plus Olaratumab vs Doxorubicin Plus Placebo 
on Survival in Patients With Advanced Soft Tissue Sarcomas: The ANNOUNCE Randomized Clinical Trial. JAMA (2020) 323(13):1266-76. doi: 10.1001/jama.2020.1707

14. Ray-Coquard IL, Domont J, Tresch-Bruneel E, Bompas E, Cassier PA, Mir O, et al. Paclitaxel Given Once Per Week With or Without Bevacizumab in Patients With Advanced Angiosarcoma: A Randomized Phase II Trial. J Clin Oncol (2015) 33(25):2797-802. doi: 10.1200/JCO.2015.60.8505

15. Martin-Broto J, Pousa AL, de Las Penas R, Garcia Del Muro X, Gutierrez A, Martinez-Trufero J, et al. Randomized Phase II Study of Trabectedin and Doxorubicin Compared With Doxorubicin Alone as First-Line Treatment in Patients With Advanced Soft Tissue Sarcomas: A Spanish Group for Research on Sarcoma Study. J Clin Oncol (2016) 34(19):2294-302. doi: 10.1200/ JCO.2015.65.3329

16. Pollack SM, Redman MW, Baker KK, Wagner MJ, Schroeder BA, Loggers ET, et al. Assessment of Doxorubicin and Pembrolizumab in Patients With Advanced Anthracycline-Naive Sarcoma: A Phase 1/2 Nonrandomized Clinical Trial. JAMA Oncol (2020) 6(11):1778-82. doi: 10.1001/ jamaoncol.2020.3689

17. Folkman J. Antiangiogenesis in Cancer Therapy-Endostatin and its Mechanisms of Action. Exp Cell Res (2006) 312(5):594-607. doi: 10.1016/ j.yexcr.2005.11.015

18. Jain RK. Antiangiogenesis Strategies Revisited: From Starving Tumors to Alleviating Hypoxia. Cancer Cell (2014) 26(5):605-22. doi: 10.1016/ j.ccell.2014.10.006

19. Qin S, Li A, Yi M, Yu S, Zhang M, Wu K. Recent Advances on AntiAngiogenesis Receptor Tyrosine Kinase Inhibitors in Cancer Therapy. J Hematol Oncol (2019) 12(1):27. doi: 10.1186/s13045-019-0718-5

20. O'Reilly MS, Boehm T, Shing Y, Fukai N, Vasios G, Lane WS, et al. Endostatin: An Endogenous Inhibitor of Angiogenesis and Tumor Growth. Cell (1997) 88 (2):277-85. doi: 10.1016/s0092-8674(00)81848-6

21. Li T, Kang G, Wang T, Huang H. Tumor Angiogenesis and Anti-Angiogenic Gene Therapy for Cancer. Oncol Lett (2018) 16(1):687-702. doi: 10.3892/ ol.2018.8733

22. Ling Y, Yang Y, Lu N, You QD, Wang S, Gao Y, et al. Endostar, a Novel Recombinant Human Endostatin, Exerts Antiangiogenic Effect via Blocking VEGF-Induced Tyrosine Phosphorylation of KDR/Flk-1 of Endothelial Cells. Biochem Biophys Res Commun (2007) 361(1):79-84. doi: 10.1016/ j.bbrc.2007.06.155

23. Han B, Xiu Q, Wang H, Shen J, Gu A, Luo Y, et al. A Multicenter, Randomized, Double-Blind, Placebo-Controlled Study to Evaluate the Efficacy of Paclitaxel-Carboplatin Alone or With Endostar for Advanced non-Small Cell Lung Cancer. J Thorac Oncol (2011) 6(6):1104-9. doi: 10.1097/ JTO.0b013e3182166b6b

24. Cui C, Mao L, Chi Z, Si L, Sheng X, Kong Y, et al. A Phase II, Randomized, Double-Blind, Placebo-Controlled Multicenter Trial of Endostar in Patients With Metastatic Melanoma. Mol Ther (2013) 21(7):1456-63. doi: 10.1038/ mt.2013.79

25. Jin T, Li B, Chen XZ. A Phase II Trial of Endostar Combined With Gemcitabine and Cisplatin Chemotherapy in Patients With Metastatic Nasopharyngeal Carcinoma (NCT01612286). Oncol Res (2013) 21(6):31723. doi: $10.3727 / 096504014 X 13983417587401$

26. Lu S, Li L, Luo Y, Zhang L, Wu G, Chen Z, et al. A Multicenter, Open-Label, Randomized Phase II Controlled Study of Rh-Endostatin (Endostar) in Combination With Chemotherapy in Previously Untreated Extensive-Stage Small-Cell Lung Cancer. J Thorac Oncol (2015) 10(1):206-11. doi: 10.1097/ JTO.0000000000000343

27. Huang W, Liu J, Wu F, Chen K, Li N, Hong Y, et al. The Efficacy and Safety of Endostar Combined With Taxane-Based Regimens for HER-2-Negative Metastatic Breast Cancer Patients. Oncotarget (2016) 7(21):31501-7. doi: 10.18632/oncotarget.8967

28. Chen J, Yao Q, Huang M, Wang B, Zhang J, Wang T, et al. A Randomized Phase III Trial of Neoadjuvant Recombinant Human Endostatin, Docetaxel and Epirubicin as First-Line Therapy for Patients With Breast Cancer (CBCRT01). Int J Cancer (2018) 142(10):2130-8. doi: 10.1002/ijc.31217

29. Jin T, Jiang F, Jin Q-F, Piao Y-F, Chen X-Z. Endostar Combined With Gemcitabine and Cisplatin Chemotherapy for Patients With Metastatic Nasopharyngeal Carcinoma: An Update. Transl Oncol (2018) 11(2):286-91. doi: 10.1016/j.tranon.2018.01.002

30. Li Y, Tian Y, Jin F, Wu W, Long J, Ouyang J, et al. A Phase II Multicenter Randomized Controlled Trial to Compare Standard Chemoradiation With or Without Recombinant Human Endostatin Injection (Endostar) Therapy for the Treatment of Locally Advanced Nasopharyngeal Carcinoma: Long-Term Outcomes Update. Curr Probl Cancer (2020) 44(1):100492. doi: 10.1016/ j.currproblcancer.2019.06.007

31. Ma Z, Guo L, Cui X, Liu H, Liu L. Rh-Endostatin Concomitant With Chemotherapy Versus Single Agent Chemotherapy for Treating Soft Tissue and Bone Sarcomas: A Systematic Review and Meta-Analysis. J Pharm Pharm Sci (2018) 21(1):386-97. doi: 10.18433/jpps30034

32. Zhang LP, Liao XY, Xu YM, Yan LJ, Yan GF, Wang XX, et al. Efficacy and Safety of Endostar(R) Combined With Chemotherapy in Patients With Advanced Soft Tissue Sarcomas. Asian Pac J Cancer Prev (2013) 14 (7):4255-9. doi: 10.7314/apjcp.2013.14.7.4255

33. Xing P, Zhang J, Yan Z, Zhao G, Li X, Wang G, et al. Recombined Humanized Endostatin (Endostar) Combined With Chemotherapy for Advanced Bone and Soft Tissue Sarcomas in Stage IV. Oncotarget (2017) 8(22):36716-27. doi: 10.18632/oncotarget.13545

34. Li K, Shi M, Qin S. Current Status and Study Progress of Recombinant Human Endostatin in Cancer Treatment. Oncol Ther (2018) 6(1):21-43. doi: 10.1007/ s40487-017-0055-1

35. Izzedine H, Ederhy S, Goldwasser F, Soria JC, Milano G, Cohen A, et al. Management of Hypertension in Angiogenesis Inhibitor-Treated Patients. Ann Oncol (2009) 20(5):807-15. doi: 10.1093/annonc/mdn713

36. Wu S, Kim C, Baer L, Zhu X. Bevacizumab Increases Risk for Severe Proteinuria in Cancer Patients. J Am Soc Nephrol (2010) 21(8):1381-9. doi: 10.1681/ASN.2010020167

37. Cortes J, Calvo V, Ramírez-Merino N, O'Shaughnessy J, Brufsky A, Robert N, et al. Adverse Events Risk Associated With Bevacizumab Addition to Breast Cancer Chemotherapy: A Meta-Analysis. Ann Oncol (2012) 23(5):1130-7. doi: $10.1093 /$ annonc/mdr432

38. Shao F, Zhang H, Yang X, Luo X, Liu J. Adverse Events and Management of Apatinib in Patients With Advanced or Metastatic Cancers: A Review. Neoplasma (2020) 67(4):715-23. doi: 10.4149/neo_2020_190801N701

39. Riedel RF. Systemic Therapy for Advanced Soft Tissue Sarcomas: Highlighting Novel Therapies and Treatment Approaches. Cancer (2012) 118(6):1474-85. doi: 10.1002/cncr.26415

Conflict of Interest: The authors declare that the research was conducted in the absence of any commercial or financial relationships that could be construed as a potential conflict of interest.

Publisher's Note: All claims expressed in this article are solely those of the authors and do not necessarily represent those of their affiliated organizations, or those of the publisher, the editors and the reviewers. Any product that may be evaluated in this article, or claim that may be made by its manufacturer, is not guaranteed or endorsed by the publisher.

Copyright (c) 2022 Liao, Zhang, Yang, Liu, Yang, Li, Xing, Teng, Yang, Zhao, Zhao, Bai, Zhu and Yang. This is an open-access article distributed under the terms of the Creative Commons Attribution License (CC BY). The use, distribution or reproduction in other forums is permitted, provided the original author(s) and the copyright owner(s) are credited and that the original publication in this journal is cited, in accordance with accepted academic practice. No use, distribution or reproduction is permitted which does not comply with these terms. 